\title{
EXISTENCE OF GENERALIZED HOMOCLINIC ORBITS FOR ONE PARAMETER FAMILIES OF FLOWS
}

\author{
KONSTANTIN MISCHAIKOW
}

(Communicated by George R. Sell)

\begin{abstract}
We consider a one parameter family of flows generated by $\dot{x}=$ $f(x, \lambda)$, where $\lambda \in[0,1]$. We, also, assume that there exists an isolated invariant set, $S$, which continues across the interval $[0,1]$, and that we know the connection matrices for $\dot{x}=f(x, 1)$ and $\dot{x}=f(x, 0)$. We then give conditions under which a Morse decomposition of $S$ cannot continue across $[0,1]$. Furthermore, using the language of chain recurrence we define a generalized homoclinic orbit and give conditions under which such objects exist.
\end{abstract}

Introduction. Often, when dealing with a parametrized family of differential equations, $\dot{x}=f(x, \lambda)$, one has an understanding of the system for various parameter values, say $\lambda=0,1$. The question then arises, what can one say about the behavior of solutions for the parameter values $\lambda \in(0,1)$ ? In [7], Reineck discusses this problem under the assumptions that one has an isolated invariant set and a Morse decomposition, thereof, which continue across the interval $[0,1]$. There are, however, many examples for which these hypotheses are too strong. The purpose of this paper is to give conditions under which the Morse decomposition cannot continue across the interval and then describe the behavior of solutions at these global bifurcation points.

The archetype of such behavior is the following example. Let $c:[0,1] \rightarrow \mathbf{R}^{n}$. Assume that $c(\lambda)$ is a hyperbolic critical point for each value of $\lambda$, then $c(\lambda)$ is an isolated invariant set. Now assume that for $\lambda=0,1, c(\lambda)$ is also a Morse set for the global isolated invariant set, but that for a unique value, $\bar{\lambda} \in(0,1), c(\bar{\lambda})$ is the critical point for a homoclinic orbit. In this case $c(\bar{\lambda})$ cannot be a Morse set of the global isolated invariant set and hence the original Morse decomposition at $\lambda=1$ cannot be continued across $[0,1]$.

The techniques we shall employ involve the Conley index, the connection matrix, and transition systems. The type of assumptions we make can be roughly stated as follows. There exists an isolated invariant set $S^{\lambda}$ of $\dot{x}=f(x, \lambda)$ which continues across the interval $[0,1]$, we know the connection matrix associated with $S^{0}, S^{1}$, and the transition system from $S^{1}$ to $S^{0}, c:[0,1] \rightarrow \mathbf{R}^{n}$ is a parametrized family of hyperbolic critical points associated with a repeller (or an attractor), and finally the connection matrix entry describing connections from $c(1)$ to $c(0)$ in the transition system is 0 . The conclusion we would like to draw is that there exists a value $\bar{\lambda}$ at which there exists a homoclinic orbit to $c(\bar{\lambda})$. This, however, is unrealistic since we

Received by the editors December 9, 1986.

1980 Mathematics Subject Classification (1985 Revision). Primary 34C35, 58F 14.

This research was supported in part by ARO under contract number DAAG-29-83-K-0029 and the AFOSR under grants numbered AFOSR-81-0116-C and AFOSR-84-0376. 
are only making global assumptions and, hence, the behavior of the vector field can be changed in a neighborhood of any one orbit without affecting the hypothesis. Thus, we can only conclude that there exists a generalized homoclinic orbit, where this orbit is defined in terms of the chain recurrent set for $\dot{x}=f(x, \bar{\lambda})$.

In $\S 1$ we introduce the notation and basic concepts which will be used throughout this paper. Since this is just a cursory review we expect the reader to be familiar with the Conley index (see $[\mathbf{1}, \mathbf{8}, \mathbf{9}]$ ), the continuation of isolated invariants sets $[\mathbf{8}]$, the connection matrix $[\mathbf{3}, \mathbf{4}, \mathbf{7}]$ and transition systems $[\mathbf{7}]$.

In $\S 2$ we obtain specific results concerning the connection matrices for transition systems in which the Morse decomposition continues across the interval. This relies to a large extent on the results of [7].

$\S 3$ discusses chain recurrence and introduces the concept of a generalized homoclinic orbit. Most of the results can be found in [1 or 2].

Finally, $\S 4$ contains the theorems concerning the noncontinuation of the Morse decomposition and the existence of generalized homoclinic orbits for some parameter value. We also discuss some applications of these theorems.

1. Notation. Throughout this paper $(X, d)$ will denote a locally compact metric space with metric $d$. If $A \subset X$ then $\operatorname{cl}(A)$ denotes the closure of $A$ and $\partial A$ denotes the boundary of $A$. A flow on $X$ means a continuous map $\varphi: X \times \mathbf{R} \rightarrow X$ such that $\varphi_{0}(x)=x$ and $\varphi_{t}\left(\varphi_{s}(x)\right)=\varphi_{t+s}(x)$. If $I \subset \mathbf{R}$ and $A \subset X$ then $\varphi_{I}(\dot{A})=\varphi(A \times I)$. We define

$$
\omega(A)=\bigcap_{t>0} \operatorname{cl}\left(\varphi_{[t, \infty)}(A)\right) \quad \text { and } \quad \omega^{*}(A)=\bigcap_{t<0} \operatorname{cl}\left(\varphi_{(-\infty, t]}(A)\right) .
$$

$N \subset X$ will always denote a compact subset. Let $\not{H}$ denote the set of closed subsets of $N$. For $F, G \in \mathcal{H}$ set

$$
\bar{\rho}(F, G)=\inf \{\varepsilon \mid F \text { is contained in an } \varepsilon \text {-neighborhood of } G\} \text {. }
$$

Define $\rho(F, G)=\max \{\bar{\rho}(F, G), \bar{\rho}(G, F)\}$. Then $(\mathcal{H}, \rho)$ is a compact metric space. The metric $\rho$ is referred to as the Hausdorff metric.

$S \subset X$ is called invariant if $\varphi_{\mathbf{R}}(S)=S$. The maximal invariant subset of $N \subset X$ is denoted by $I(N)=\left\{x \in X \mid \varphi_{\mathbf{R}}(x) \subset N\right\}$. If $S=I(N) \subset \operatorname{int} N$ then $S$ is called an isolated invariant set with isolating neighborhood $N$. A compact invariant set $A \subset S$ is called an attractor in $S$ if there exists a neighborhood $U$ of $A$ in $S$ such that $A=\omega(U)$. A compact invariant set $A^{*} \subset S$ is said to be a repeller in $S$ if there exists a neighborhood $U$ of $A^{*}$ in $S$ such that $\omega^{*}(U)=A^{*}$. Given an attractor $A$ in $S, A^{*}=\{x \in S \mid \omega(x) \cap A=\varnothing\}$ is the complementary repeller of $A$ in $S$. We call $\left(A, A^{*}\right)$ an attractor-repeller pair.

A partially ordered set $(P,>)$ consists of a set $P$ and a relation, $>$, satisfying:

(a) $i>i$ never holds for $i \in P$.

(b) If $i>j$ and $j>k$ then $i>k$ for all $i, j, k \in P$.

If $K$ and $K^{\prime}$ are invariant sets then the set of connections from $K$ to $K^{\prime}$ is given by

$$
C\left(K, K^{\prime}\right)=\left\{x \in X \mid \omega(x) \subset K^{\prime} \text { and } \omega^{*}(x) \subset K\right\} .
$$

Given an isolated invariant set $S$, a Morse decomposition of $S$ is a finite collection $\mathcal{M}(S)=\{M(i) \mid i \in(P,>)\}$ of mutually disjoint compact invariant sets in $S$, indexed by $P$, such that if $x \in S$ then $x \in M(i)$ or $x \in C(M(i), M(j))$ where $i>j$. The 
individual sets $M(i)$ are called Morse sets and are themselves isolated invariant sets.

An interval in $(P,>)$ is a subset, $I \subset P$, for which $i, j \in I$ and $i>k>j$ implies that $k \in I$. Define

$$
M(I)=\left\{x \mid \omega(x) \subset M(i) \text { and } \omega^{*}(x) \subset M(j) \text { for } i, j \in I\right\} .
$$

It is easy to check that $M(I)$ is an isolated invariant set and, hence, the Conley index $h(M(I))$ is defined. We shall be interested in $H_{*}(h(M(I)) ; \mathbf{F})$ where $\mathbf{F}$ is a field. To simplify the notation we write $H(I)=H_{*}(h(M(I)) ; \mathbf{F})$ and $H_{k}(I)=$ $H_{k}(h(M(I)) ; \mathbf{F})$. In particular, if $I=\{i\}$ a single element of $P$ we write $H(i)=$ $H_{*}(h(M(i)) ; \mathbf{F})$ and $H_{k}(i)=H_{k}(h(M(i)) ; \mathbf{F})$.

For a definition of connection matrices the reader is referred to $[\mathbf{3}, \mathbf{4}$, or $\mathbf{7}]$. We will, however, state some of the properties satisfied by connection matrices. To do so, we need to introduce some more notation. Let $\Delta: \bigoplus_{i \in P} H(i) \rightarrow \bigoplus_{i \in P} H(i)$ be a matrix. Then $\Delta=[\Delta(i, j)], i, j \in P$, where $\Delta(i, j): H(j) \rightarrow H(i)$. Let 0 denote the zero matrix. We call $\Delta$ a degree -1 boundary map if $\Delta \circ \Delta=0$ and $\Delta(i, j)\left(H_{k}(j)\right) \subset H_{k-1}(i)$. Define

$$
\Delta_{k}=\left.\Delta\right|_{\oplus_{i \in P} H_{k}(i)} .
$$

If $\Delta$ is a degree -1 boundary map then the image of $\Delta_{k+1}$, denoted $\operatorname{Im} \Delta_{k+1}$, is contained in the kernel of $\Delta_{k}$, denoted by $\operatorname{Ker} \Delta_{k}$. Thus we can define $H_{n} \Delta=$ $\operatorname{Ker} \Delta_{k} / \operatorname{Im} \Delta_{k+1}$. Finally, if $I$ is an interval in $P$, let $\Delta(I)=[\Delta(i, j)]$ where $i, j \in I$,

$$
\Delta_{k}(I)=\left.\Delta(I)\right|_{\oplus_{i \in I} H_{k}(i)}, \quad \text { and } \quad H_{k} \Delta(I)=\operatorname{Ker} \Delta_{k}(I) / \operatorname{Im} \Delta_{k}(I)
$$

REMARK 1.1. If $\Delta: \bigoplus_{i \in P} H(i) \rightarrow \bigoplus_{i \in P} H(i)$ is a connection matrix associated with the Morse decomposition $\mathcal{M}(S)=\{M(i) \mid i \in(P,>)\}$ of $S$ then $\Delta$ satisfies the following four properties:

(a) If $i \ngtr j$ then $\Delta(j, i)=0$.

(b) $\Delta$ is a degree -1 boundary map.

(c) For every interval $I \subset P, H_{k} \Delta(I) \approx H_{k}(I)$.

(d) $\Delta(I)$ is a connection matrix for the Morse decomposition $\mathcal{M}(M(I))=\{M(i) \mid i$ $\in I \subset(P,>)\}$ of $M(I)$.

On occasion it will be more convenient to write the connection matrix entries in terms of the Morse sets instead of the indices of the Morse sets, i.e. $\Delta(M(i), M(j))=$ $\Delta(i, j)$, etc. This should not lead to any confusion.

Another property of a connection matrix is that if $\left(A, A^{*}\right)$ is an attractor-repeller pair in $S$, then the following is a long exact sequence.

$$
\begin{aligned}
\cdots & \rightarrow H_{n}(h(A) ; \mathbf{F}) \rightarrow H_{n}(h(S) ; \mathbf{F}) \\
& \rightarrow H_{n}\left(h\left(A^{*}\right) ; \mathbf{F}\right)^{\Delta_{n}} \stackrel{\left(A^{*}, A\right)}{\rightarrow} H_{n-1}(h(A) ; \mathbf{F}) \rightarrow \cdots .
\end{aligned}
$$

For any collection of Morse sets $\{M(i) \mid i \in P\}$ there is a minimal partial order on $P$ defined by taking the transitive closure of the relations $i>j$ if $C(M(i), M(j)) \neq$ $\varnothing$. This partial order is called the flow defined partial order on $P$.

As was mentioned in the introduction we are interested in analyzing a parametrized family of differential equations

$$
\dot{x}=f(x, \lambda)
$$


where $x \in X$ and $\lambda \in \Lambda=[0,1]$. For technical reasons, however, we assume that the parametrized family extends over $\Lambda_{2 \theta}=[-2 \theta, 1+2 \theta]$ where $0<\theta<<1$. Let $\Lambda_{\theta}=[-\theta, 1+\theta]$. For every $\lambda \in \Lambda_{2 \theta}$, equation (1.2) generates a flow denoted by $\varphi^{\lambda}$. We can now define a flow $\Phi$ on $X \times \Lambda_{2 \theta}$ by $\Phi_{t}(x, \lambda)=\left(\varphi_{t}^{\lambda}(x), \lambda\right)$. Let $\eta_{x}: X \times \Lambda_{2 \theta} \rightarrow X$ and $\pi_{\Lambda}: X \times \Lambda_{2 \theta} \rightarrow \Lambda_{2 \theta}$ be the canonical projective maps. Define $\Lambda(N)=\left\{\lambda \in \Lambda_{2 \theta} \mid N \times \lambda\right.$ is an isolating neighborhood in $X \times \lambda$ under $\left.\varphi^{\lambda}\right\}$. Define $S=\left\{S^{\lambda} \mid \lambda \in \Lambda_{2 \theta}, S^{\lambda} \subset X\right.$ compact, $S^{\lambda}$ is an isolated invariant set in $X \times \lambda$ under $\left.\varphi^{\lambda}\right\}$. For every compact set $N \subset X$ define $\sigma_{N}: \Lambda(N) \rightarrow S$ by $\sigma_{N}(\lambda)=I(N \times \lambda)$. The topology on $S$ is generated by the sets $\left\{\sigma_{N}(U) \mid N \subset X\right.$ compact, $U \subset \Lambda(N)$ open\}.

We say that $S^{\lambda_{0}}$ and $S^{\lambda_{1}}$ are related by continuation if there exists a continuous map $\sigma:\left[\lambda_{0}, \lambda_{1}\right] \rightarrow S$ such that $\sigma\left(\lambda_{0}\right)=S^{\lambda_{0}}, \sigma\left(\lambda_{1}\right)=S^{\lambda_{1}}$ and $\pi_{\Lambda} \circ \sigma=1_{\Lambda}$. On the other hand, given $S^{\lambda_{1}}$ and the map $\sigma$ we say that $S^{\lambda_{1}}$ continues across the interval $\left[\lambda_{0}, \lambda_{1}\right]$.

2. Transition systems. We now turn to a definition and discussion of transition systems, their connection matrices, and their relationship to the flow, $\Phi$, defined in §1. A general reference for these systems is Reineck [7] however we shall extend his results to a slightly more general global isolated invariant set.

We begin with a continuous map $\sigma: \Lambda_{2 \theta} \rightarrow S$ satisfying $\pi_{\Lambda} \circ \sigma=1_{\Lambda}$. Define $S=\bigcup_{\lambda \in \Lambda} \sigma(\lambda)$. Then $S$ is an isolated invariant set in $X \times \Lambda$ under $\Phi$ (see [8, Lemma 6.4(i)]). Choose $N \subset X \times \Lambda_{\theta}$ such that $\pi_{\Lambda}(N)=\Lambda_{\theta}$ and $I(N)=\bigcup_{\lambda \in \Lambda_{\theta}} \sigma(\lambda)$ under $\Phi$ restricted to $X \times \Lambda_{\theta}$.

Consider the transition system defined by

$$
\dot{x}=f(x, \lambda), \quad \dot{\lambda}=\mu_{n} \lambda(\lambda-1)
$$

where $\mu_{n}>\mu_{n+1}$ and $\mu_{n} \rightarrow 0$ as $n \rightarrow \infty$. This system generates a flow which we denote by $\Phi^{n}$. Notice that $\Phi^{n}$ is defined on $X \times \Lambda_{2 \theta}$. The following lemma is easily checked.

LEMMA 2.1. $\sigma(0)$ and $\sigma(1)$ are isolated invariant sets under $\Phi_{t}^{n}$.

Given two invariant sets $K, K^{\prime}$ and the flow $\Phi^{n}$, define $C_{n}\left(K, K^{\prime}\right)=\{z \mid \omega(z) \subset$ $K^{\prime}, \omega^{*}(z) \subset K$ and $\left.\Phi_{\mathbf{R}}^{n}(z) \subset N\right\}$. Clearly $C_{n}\left(K, K^{\prime}\right)$ depends on the flow $\Phi_{t}^{n}$. Define

$$
S_{n}=\sigma(0) \cup \sigma(1) \cup C_{n}(\sigma(1), \sigma(0)) .
$$

Proposition 2.2. For $n$ sufficiently large, $N$ is an isolating neighborhood for $S_{n}$ under the flow $\Phi^{n}$.

PrOOF. The proof is by contradiction, thus we can assume that $N$ is not an isolating neighborhood for all $S_{n}$. It follows directly from $\left(\tau_{n}\right)$ that $\pi_{\Lambda}(I(N)) \subset$ $[0,1]$. Furthermore, $I(N) \cap(X \times i)=\sigma(i)$ for $i=0,1$ by Lemma 2.1. Thus $I(N) \subset S_{n}$ for ever flow $\Phi^{n}$. Since we are assuming $I(N)$ is not an isolating neighborhood, for every $n$ there exists $\left(x_{n}, \lambda_{n}\right) \in \partial N \cap S_{n}$. Given the flow $\Phi^{n}$ let

$$
\bar{c}_{n}=\operatorname{cl}\left(\Phi_{\mathbf{R}}^{n}\left(x_{n}, \lambda_{n}\right) \cup \omega\left(x_{n}, \lambda_{n}\right) \cup \omega^{*}\left(x_{n}, \lambda_{n}\right)\right) .
$$

Let $\bar{c}$ denote the limit of a convergent subsequence of $\left\{\bar{c}_{n}\right\}$ under the Hausdorff metric. By [7, Lemma 3.6], $\bar{c}$ is compact, connected and invariant under $\Phi$. But $\bar{c} \cap \partial N \neq \varnothing$ hence $S \cap \partial N \neq \varnothing$ which contradicts the definition of $N$. 
From now on $\mu_{1}$ is chosen sufficiently small so that $N$ isolates $S_{n}$ under $\Phi^{n}$ for all $n$.

PROPOSITION 2.3. $h\left(S_{n}\right) \sim \overline{0}$.

PROOF. By perturbing the transition system $\left(\tau_{n}\right)$ we shall show that $h\left(S_{n}\right) \sim$ $h(\varnothing) \sim \overline{0}$. Define the system

$$
\left(\tau_{n}(\xi)\right) \quad \dot{x}=f(x, \lambda), \quad \dot{\lambda}=\mu_{n}(\lambda-\xi)(\lambda-1) .
$$

Denote the flow generated by $\tau_{n}(\xi)$ by $\Phi^{n \xi}$. Notice that $\sigma(\xi)$ and $\sigma(1)$ are isolated invariant sets under $\Phi^{n \xi}$. Define

$$
C_{n}(\sigma(1), \sigma(\xi))=\left\{z \mid \omega(z) \subset \sigma(\xi), \omega^{*}(z) \subset \sigma(1), \text { and } \Phi_{\mathbf{R}}^{n \xi}(z) \subset N\right\} .
$$

Let $S_{n}[\xi, 1]=\sigma(\xi) \cup \sigma(1) \cup C_{n}(\sigma(1), \sigma(\xi))$.

From Proposition 2.2 there exists $N$ an isolating neighborhood for $S_{n}[0,1]$ which also isolates $\bigcup_{\lambda \in \Lambda_{\theta}} \sigma(\lambda)$ in $X \times \Lambda_{\theta}$. By [8, Lemma 6.2] we can assume that

$$
N=\bigcup_{i=1}^{I} N\left(\lambda_{i}, \lambda_{i+2}\right)
$$

where $N\left(\lambda_{i}, \lambda_{i+2}\right)=N_{i} \times\left[\lambda_{i}, \lambda_{i+2}\right]$ for some compact set $N_{i} \subset X, \lambda_{i}<\lambda_{i+1}<$ $\lambda_{i+2}, \lambda_{1}=-\theta, \lambda_{I+2}=I+\theta, \lambda_{2}>0$ and $\lambda_{I+1}<1$. Finally let $\lambda_{i}<\eta_{i}<\lambda_{i+1}$.

One can now easily check that if $0 \leq \xi<\lambda_{3}$ then $\bigcup_{i=1}^{I} N\left(\lambda_{i}, \lambda_{i+2}\right)$ is an isolating neighborhood for $S_{n}[\xi, 1]$. Thus $h\left(S_{n}[0,1]\right) \sim h\left(S_{n}\left[\eta_{2}, 1\right]\right)$. Similarly, for $\lambda_{2}<\xi<\lambda_{4}, \bigcup_{i=2}^{I} N\left(\lambda_{i}, \lambda_{i+2}\right)$ is an isolating neighborhood for $S_{n}[\xi, 1]$. Thus $h\left(S_{n}\left[\eta_{2}, 1\right]\right) \sim h\left(S_{n}\left[\eta_{3}, 1\right]\right)$. Continuing in this manner after $I$ steps we can conclude that $h\left(S_{n}[0,1]\right) \sim h\left(S_{n}[1,1]\right)$, where $S_{n}[1,1]=\sigma(1)$ is an isolated invariant set for $\dot{x}=f(x, \lambda), \dot{\lambda}=\mu_{n}(\lambda-1)^{2}$. However, this perturbs to $\dot{x}=f(x, \lambda), \dot{\lambda}>0$ and hence $h\left(S_{n}[0,1]\right) \sim h(0)$.

THEOREM 2.4. Let $\Delta T^{n}$ be a connection matrix associated with the Morse decomposition of $S_{n}$ given by

$$
\mathcal{M}\left(S_{n}\right)=\{\sigma(0), \sigma(1) \mid \sigma(1)>\sigma(0)\} .
$$

Then $\Delta T_{k}^{n}(\sigma(1), \sigma(0))$ is an isomorphism. (Recall that $\Delta T^{n}(\sigma(1), \sigma(0))$ in the entry of $\Delta T^{n}$ denoting the map from $H(\sigma(1))$ to $H(\sigma(0))$.)

ProOF. From Proposition $2.3, H_{k}\left(h\left(S_{n}\right) ; \mathbf{F}\right)=0$ for all $k$. Now apply this to (1.1).

Though Theorem 2.4 appears to be trivial it can be easily applied to more complicated Morse decompositions which continue over $[0,1]$. To do so we will need the following lemma.

LEMMA 2.5 [8, LEMMA 6.4(III)]. Let $\alpha: \Lambda_{2 \theta} \rightarrow S$ be continuous such that $\pi_{\Lambda} \circ \sigma=1_{\Lambda}$ and $\alpha(\lambda)$ is an attractor in $\sigma(\lambda)$. Furthermore, let $\alpha^{*}(\lambda)$ denote the complementary repeller of $\alpha(\lambda)$ in $\sigma(\lambda)$. Then $\alpha^{*}: \Lambda_{2 \theta} \rightarrow S$ is continuous and the sets $A=\bigcup_{\lambda \in \Lambda} \alpha(\lambda), A^{*}=\bigcup_{\lambda \in \Lambda} \alpha^{*}(\lambda)$ form an attractor-repeller pair in $S$.

Proposition 2.6. Let $\alpha$ and $\alpha^{*}$ be a defined as above. Let

$$
\mathcal{M}\left(S_{n}\right)=\left\{\alpha(1), \alpha^{*}(1), \alpha(0), \alpha^{*}(0)\right\} .
$$


Then for $n$ sufficiently large, $\alpha^{*}(0)$ and $\alpha^{*}(1)$ are adjacent under the flow defined partial order. The same holds true for $\alpha(0)$ and $\alpha(1)$.

PROOF. Since both cases are proved in a similar manner we shall only show that $\alpha(0)$ and $\alpha(1)$ are adjacent. From $\left(\tau_{n}\right)$ it is clear that in the flow defined partial order $\alpha(0) \ngtr \alpha(1)$. By the definition of $\alpha$ and $\alpha^{*}, \alpha(i) \ngtr \alpha^{*}(i), i=0,1$, thus $\alpha(0)$ and $\alpha(1)$ are adjacent unless $\alpha(1)>\alpha^{*}(0)>\alpha(0)$. So assume this is the case. Then under $\Phi^{n}$ there exists a connection from $\alpha(1)$ to $\alpha^{*}(0)$. Hence by [7, Theorem 3.13] there exists $\bar{\lambda}$ such that $\alpha(\bar{\lambda})>\alpha^{*}(\bar{\lambda})$ under the flow $\varphi^{\bar{\lambda}}$. This contradicts the definition of $\alpha$ and $\alpha^{*}$.

We now know that under the flow $\Phi^{n}, A_{n}=\alpha(0) \cup \alpha(1) \cup C_{n}(\alpha(1), \alpha(0))$ and $A_{n}^{*}=\alpha^{*}(0) \cup \alpha^{*}(1) \cup C_{n}\left(\alpha^{*}(1), \alpha^{*}(0)\right)$ are isolated invariant sets. Repeating what we did for $S$ we can let $\mathcal{M}\left(A_{n}\right)=\{\alpha(1), \alpha(0)\}$ be a Morse decomposition for $A_{n}$ and $\mathcal{M}\left(A_{n}^{*}\right)=\left\{\alpha^{*}(1), \alpha^{*}(0)\right\}$ be a Morse decomposition for $A_{n}^{*}$.

Let $\Delta T^{n}$ denote a connection matrix associated with $\mathcal{M}\left(S_{n}\right)$ as defined in Proposition 2.6. Then we can write

$$
\begin{gathered}
\alpha(0) \\
\Delta T^{*}(0) \\
\alpha(0) \\
\alpha^{*}(0) \\
\alpha(1) \\
\alpha^{*}(1)
\end{gathered}\left[\begin{array}{cccc}
0 & \mathbf{E} & \mathbf{F}_{11} & \mathbf{F}_{12} \\
0 & 0 & \mathbf{F}_{21} & \mathbf{F}_{22} \\
0 & 0 & 0 & \mathbf{G} \\
0 & 0 & 0 & 0
\end{array}\right]
$$

PROPOSITION 2.7. Let $\Delta A^{n}$ and $\Delta A^{n}$ be connection matrices associated with $\mathcal{M}\left(A_{n}\right)$ and $\mathcal{M}\left(A_{n}^{*}\right)$ respectively. Then:

(i) $\mathbf{F}_{11}=\Delta A^{n}(\alpha(1), \alpha(0))$ and $\mathbf{F}_{22}=\Delta A^{n^{*}}\left(\alpha^{*}(1), \alpha^{*}(0)\right)$.

(ii) $\Delta A_{k}^{n}(\alpha(1), \alpha(0))$ and $\Delta A_{k}^{n^{*}}\left(\alpha^{*}(1), \alpha^{*}(0)\right)$ are isomorphisms.

PROOF. (i) This follows from Proposition 2.6 which shows that $\left\{\alpha^{*}(0), \alpha^{*}(1)\right\}$ are adjacent, as are $\{\alpha(0), \alpha(1)\}$ in $\mathcal{M}\left(S_{n}\right)$.

(ii) This is a restatement of Theorem 2.4.

EXAMPLE 2.8. Let $\sigma, \alpha, \alpha^{*}: \Lambda_{2 \theta} \rightarrow S$ be given as above. Assume that there exists $\beta, \beta^{*}: \Lambda_{2 \theta} \rightarrow S$ such that $\beta(\lambda)$ is an attractor in $\alpha(\lambda)$ and $\beta^{*}(\lambda)$ is the complementary repeller of $\beta(\lambda)$ in $\alpha(\lambda)$. Furthermore, assume for $\lambda \in \Lambda_{2 \theta}$ that $\beta^{*}(\lambda)$ is a hyperbolic critical point for $\varphi^{\lambda}$ with $h\left(\beta^{*}(\lambda)\right) \sim \Sigma^{l}$. We then have a Morse decomposition of $S_{n}$ given by $\mathcal{M}\left(S_{n}\right)=\left\{\alpha^{*}(1), \beta^{*}(1), \beta(1), \alpha^{*}(0), \beta^{*}(0), \beta(0)\right\}$ with an associated connection matrix $\Delta T^{n}$. Let $\mathbf{F}=\mathbf{Z}_{2}$. Then

$$
\Delta T_{k}^{n}\left(\beta^{*}(1), \beta^{*}(0)\right)= \begin{cases}1 & \text { if } k=l+1 \\ 0 & \text { otherwise }\end{cases}
$$

Perhaps it is more enlightening to consider this example in a concrete setting. Consider the 2-dimensional flow defined by $\dot{x}=y, \dot{y}=-(\lambda+1) y-f^{\prime}(x)$ where $f^{\prime}(x)=x\left(x-\frac{1}{3}\right)(x-1)$ and $\lambda \in[0,1]$. If one defines $H(x, y)=y^{2}+f(x)$ then $\dot{H}=-(\lambda+1) y^{2}$ and hence is a Lyapunov function for this system. Therefore we define $\alpha^{*}(\lambda)=\{(1,0)\}, \alpha(\lambda)=\left\{(0,0),\left(\frac{1}{3}, 0\right)\right\} \cup C\left((0,0),\left(\frac{1}{3}, 0\right)\right), \beta^{*}(\lambda)=\{(0,0)\}$ and $\beta(\lambda)=\left\{\left(\frac{1}{3}, 0\right)\right\}$. It is easy to check that for the transition system $h\left(\alpha^{*}(i)\right) \sim$ $\Sigma^{1+i}, h\left(\beta^{*}(i)\right) \sim \Sigma^{1+i}$ and $h(\beta(i)) \sim \Sigma^{i}$ where $i=0,1$. Now applying Remark 1.1, 
Theorem 2.4 and Proposition 2.7 one has that

$$
\begin{aligned}
& \beta(0) \quad \beta^{*}(0) \quad \alpha^{*}(0) \quad \beta(1) \quad \beta^{*}(1) \quad \alpha^{*}(1) \\
& \Delta T=\begin{array}{c}
\beta(0) \\
\beta^{*}(0) \\
\alpha^{*}(0) \\
\beta(1) \\
\beta^{*}(1) \\
\alpha^{*}(1)
\end{array}\left[\begin{array}{llllll}
0 & * & * & \approx & 0 & 0 \\
0 & 0 & 0 & 0 & \approx & * \\
0 & 0 & 0 & 0 & * & \approx \\
0 & 0 & 0 & 0 & * & * \\
0 & 0 & 0 & 0 & 0 & 0 \\
0 & 0 & 0 & 0 & 0 & 0
\end{array}\right]
\end{aligned}
$$

where $\approx$ denotes an isomorphism and $*$ remains to be determined.

3. Chain recurrence. This section provides a brief review of chain recurrence and its relationship to Morse decompositions. Most of what is presented here can be found in Conley [1 or 2].

Given $(x, y) \in X \times X$ and $\varepsilon, t>0$ an $(\varepsilon, t, \lambda)$ chain from $x$ to $y$ means a collection $\left\{x=x_{1}, \ldots, x_{n+1}=y ; t_{1}, \cdots, t_{n}\right\}$ such that $t_{i} \geq t$ and

$$
d\left(\varphi_{t}^{\lambda}\left(x_{i}\right), x_{i+1}\right) \leq \varepsilon, \quad \text { for all } i=1, \ldots, n \text {. }
$$

Define:

$P(\lambda)=\{(x, y) \mid$ for any $\varepsilon, t>0$ there exists an $(\varepsilon, t, \lambda)$ chain from $x$ to $y\}$.

$\Omega(K, \lambda)=\{y \in X \mid$ there exists $x \in K$ such that $(x, y) \in \mathcal{P}(\lambda)\}$.

$\Omega^{*}(K, \lambda)=\{y \in X \mid$ there exists $x \in K$ such that $(y, x) \in P(\lambda)\}$.

$R(\lambda)=\{x \mid(x, x) \in P(\lambda)\}$.

$R(\lambda)$ is called the chain recurrent set of the flow $\varphi^{\lambda}$. We can define an equivalence relation on $R(\lambda)$ by setting $x \sim y$ if and only if $(x, y) \in P(\lambda)$ and $(y, x) \in P(\lambda)$.

PROPOSITION 3.1 (CONLEY [2]). The equivalence classes of $R(\lambda)$ are precisely the components of $R(\lambda)$.

Let $\left\{E_{j}(\lambda) \mid j \in J\right\}$ denote the set of equivalence classes of $R(\lambda)$.

PROPOSITION 3.2 (CONLEY [2]). (a) $R(\lambda)$ is compact.

(b) For every $j \in J, E_{j}(\lambda)$ is compact.

(c) If $x \in E_{j}(\lambda)$ then $\omega(x) \cup \omega^{*}(x) \cup \varphi_{\mathbf{R}}^{\lambda}(x) \subset E_{j}(\lambda)$.

(d) $E_{j}(\lambda)=\Omega\left(E_{j}(\lambda), \lambda\right) \cap \Omega^{*}\left(E_{j}(\lambda), \lambda\right)$.

PROPOSITION 3.3 (CONLEY [2]). Let $S^{\lambda}$ be an isolated invariant set in $X$ under $\varphi^{\lambda}$. Let $K^{\lambda} \subset S^{\lambda}$. Then $K^{\lambda}$ is a Morse set in $S^{\lambda}$ if and only if $K=$ $\Omega(K, \lambda) \cap \Omega^{*}(K, \lambda)$ and $K \cap R(\lambda)$ is both open and closed in $R(\lambda)$.

We say that an invariant set $K$ is a chain recurrent set if for every $x, y \in K$ and every $\varepsilon, t>0$ there exists an $(\varepsilon, t, \lambda)$ chain from $x$ to $y$ which is a subset of $K$, i.e. each $x_{i}$ in the $(\varepsilon, t)$ chain is an element of $K$. Let $K$ be a compact isolated invariant chain recurrent set. Assume that $K \subset R^{\prime}(\lambda)$ a component of $R(\lambda)$. If $K \neq R^{\prime}(\lambda)$ then we say that there exists a generalized homoclinic orbit to $K$.

4. Results. We are now in a position to state and prove the major results of this paper. 
THEOREM 4.1. Let $K$ be a hyperbolic critical point under $\varphi^{\lambda}$. Assume $K$ is not a Morse set for any Morse decomposition of $\sigma(\lambda)$. Then there exists a generalized homoclinic orbit to $K$.

PROOF. The proof is by contradiction. Assume that there does not exist a generalized homoclinic orbit to $K$. Then we can set $K=E_{0}$, an equivalence class of $R(\lambda)$. By Proposition 3.2(d), $K=\Omega(K, \lambda) \cap \Omega^{*}(K, \lambda)$. By Proposition 3.3, if $K$ is both open and closed in $R(\lambda)$ then $K$ is a Morse set which is a contradiction. By Proposition 3.2(b) $K$ is closed, thus $K$ is not open in $R(\lambda)$.

Because $K$ is a hyperbolic critical point we can find an open neighborhood of $K$ on which the flow $\varphi^{\lambda}$ is topologically equivalent to

$$
\left[\begin{array}{l}
\dot{x}_{1} \\
\dot{x}_{2}
\end{array}\right]=\left[\begin{array}{cc}
I_{1} & 0 \\
0 & -I_{2}
\end{array}\right] \begin{aligned}
& x_{1} \\
& x_{2}
\end{aligned}
$$

where $I_{1}$ is the $n_{i} \times n_{i}$ identity matrix. Let $\xi=\left(x_{1}, x_{2}\right) \neq 0$. Then $K$ not open in $R(\lambda)$ implies the existence of $\left\{\xi_{n}\right\}$ such that $\xi_{n} \rightarrow 0$ as $n \rightarrow \infty$ and $\xi_{n} \in E_{j_{n}}$. Since the origin now corresponds to $E_{0}, j_{n} \neq 0$, for all $n$.

By Proposition 3.2(c), $\xi_{n}$ does not belong to the stable, $W^{\mathrm{s}}(0)$, or the unstable, $W^{\mathrm{u}}(0)$, manifold to the origin. Again by Proposition 3.2(c), $\varphi_{\mathbf{R}}^{\lambda}\left(\xi_{n}\right) \subset E_{j_{n}}$. Now from the explicit description of the flow given by 4.1 it is clear that in the Hausdorfftopology

$$
\lim _{n \rightarrow \infty}\left[\varphi_{\mathbf{R}}\left(\xi_{n}\right) \cap\left(W^{\mathrm{u}}(0) \cup W^{\mathrm{s}}(0)\right) \cap F\right] \neq 0
$$

where $F$ is a closed neighborhood of $K$. Thus by Proposition 3.2(a), $\left(W^{\mathrm{u}}(0) \cup\right.$ $\left.W^{\mathrm{s}}(0)\right) \cap R(\lambda) \neq \varnothing$. This contradicts the fact that $K=E_{0}$ is a component of $R(\lambda)$.

By a similar proof we have

PROPOSITION 4.2. Theorem 4.1 holds if $K$ is a hyperbolic periodic orbit.

Let $\sigma, \alpha$, and $\alpha^{*}$ be defined as in $\S 2$. For every $\lambda \in \Lambda$, let $\mathcal{M}(\alpha(\lambda))$ be a Morse decomposition for $\alpha(\lambda)$. Define $c: K \times \Lambda \rightarrow X \times \Lambda$, an imbedding, such that $\pi_{\Lambda} \circ c(x, \lambda)=\lambda$. To simplify the notation we will write $K(\lambda)=c(K, \lambda)$. Finally, let $\Delta T^{n}$ denote a connection matrix for $\left(\tau_{n}\right)$.

THEOREM 4.3. Assume $K(\lambda)$ satisfies the following four properties:

(i) For all $\lambda \in \Lambda, K(\lambda)$ is a compact invariant set.

(ii) For all $\lambda \in \Lambda$, there exists $M\left(p^{\lambda}\right) \in \mathcal{M}(\alpha(\lambda))$ such that $K(\lambda) \subset M\left(p^{\lambda}\right)$.

Furthermore, if $i=0,1$ then $K(i)=M\left(p^{i}\right)$ and $h(K(0)) \sim h(K(1))$.

(iii) If $M\left(i^{\lambda}\right) \in \mathcal{M}(\alpha(\lambda)), i^{\lambda} \neq p^{\lambda}$ then $C\left(M\left(i^{\lambda}\right), M\left(p^{\lambda}\right)\right)=\varnothing$.

(iv) There exists $k$ such that $\Delta T_{k}^{n}\left(p^{1}, p^{0}\right)$ is not an isomorphism. Then, there exists $\bar{\lambda} \in(0,1)$ such that $K(\bar{\lambda})$ is not a Morse set for any Morse decomposition of $\sigma(\bar{\lambda})$.

Proof. First, from (ii) we can restrict our attention to $A \subset S$, i.e. for each $\lambda$ we need only consider $\alpha(\lambda)$. So assume that for every $\lambda, K(\lambda)$ is a Morse set of $\alpha(\lambda)$. Now define $\beta^{*}: \Lambda \rightarrow S$ by $\beta^{*}(\lambda)=K(\lambda)$. Since $K(\lambda)$ is isolated for every $\lambda, \beta^{*}$ is continuous. By (iii), $\beta^{*}(\lambda)$ is a repeller in $\alpha(\lambda)$ for every $\lambda$. Define $\beta: \Lambda \rightarrow S$ by letting $\beta(\lambda)$ be the complementary attractor of $\beta^{*}(\lambda)$ in $\alpha(\lambda)$. Then by a variation of Lemma 2.5, $\beta$ is continuous. We are now in the situation described in Proposition 
2.6 and 2.7. Let $\Delta B^{n}$ be the connection matrix associated with $\mathcal{M}\left(\bigcup_{\lambda \in \Lambda} \beta^{*}(\lambda)\right)$. Then $\Delta B_{k}^{n}\left(\beta^{*}(1), \beta^{*}(0)\right)$ is an isomorphism. This contradicts (iv).

COROLLARY 4.4. If $K(\lambda)$ is a hyperbolic critical point or a hyperbolic periodic orbit and satisfies the hypotheses of Theorem 4.3, then there exists a generalized homoclinic orbit to $K(\bar{\lambda})$.

REMARK 4.5. It is clear from the proof that we can change the hypothesis of Theorem 4.3 as follows. Let $M\left(p^{\lambda}\right) \subset \mathcal{M}\left(\alpha^{*}(\lambda)\right)$ and assume that for $M\left(i^{\lambda}\right) \in$ $\mathcal{M}\left(\alpha^{*}(\lambda)\right), C\left(M\left(p^{\lambda}\right), M\left(i^{\lambda}\right)\right)=\varnothing$.

There are occasions in applications, where there exists at most one value, $\bar{\lambda} \in$ $(0,1)$, where $K(\lambda)$ is not a Morse set in $\sigma(\lambda)$. In this case we can change the hypothesis as follows. Let $\alpha_{1}, \alpha_{1}^{*}:(\bar{\lambda}, 1+\theta] \rightarrow S$ be attractor-repeller pairs in $\sigma(\lambda)$ for $\lambda \in(\bar{\lambda}, 1+\theta]$. Let $\alpha_{0}, \alpha_{0}^{*}:[-\theta, \bar{\lambda}] \rightarrow S$ be attractor-repeller pairs in $\sigma(\lambda)$ for $\lambda \in[-\theta, \bar{\lambda})$.

THEOREM 4.6. For $\lambda \in(\bar{\lambda}, 1]$, let $\alpha_{1}(\lambda)=K(\lambda)$. For $\lambda \in[0, \bar{\lambda})$ let $\alpha_{0}^{*}(\lambda)=$ $K(\lambda)$. Assume $h(K(0)) \sim h(K(1))$. Finally assume there exists a $k$ such that $\Delta T_{k}^{n}\left(\alpha_{1}(1), \alpha_{0}^{*}(0)\right)$ is not an isomorphism. Then, $K(\bar{\lambda})$ is not a Morse set for any Morse decomposition of $\sigma(\bar{\lambda})$.

ProOF. Again the proof is by contradiction. Assume $K(\lambda)$ is Morse set for all $\lambda \in[0,1]$. If $\alpha_{1}(1)$ and $\alpha_{0}^{*}(0)$ are adjacent then by Proposition 2.4 we have a contradiction. That $\alpha_{1}(1)$ and $\alpha_{0}^{*}(0)$ are adjacent follows directly from the fact that $\alpha_{1}$ is an attractor in $(\bar{\lambda}, 1]$ and $\alpha_{0}^{*}$ is a repeller on $[0, \bar{\lambda})$.

COROLLARY 4.7. If $K(\lambda)$ is a hyperbolic critical point or a hyperbolic periodic orbit and satisfies the hypothesis of Theorem 4.6, then there exists a generalized homoclinic orbit to $K(\bar{\lambda})$.

We conclude with remarks on the applicability of these results. Corollary 4.4 is motivated by [6] where 2-dimensional predator-prey with group defense models are considered. In these cases $K(\lambda)$ is a hyperbolic critical point. Furthermore, there are no degenerate critical points under $\varphi^{\lambda}$, thus Poincare-Bendixson is applicable. If we denote by $R^{\prime}(\bar{\lambda})$, the component of $K(\bar{\lambda})$ in $R(\bar{\lambda})$ then simple analysis of the equations shows that $K(\bar{\lambda})$ is the only critical point in $R(\bar{\lambda})$. Thus the generalized homoclinic orbit to $K(\bar{\lambda})$ is in fact a homoclinic orbit to $K(\bar{\lambda})$.

Corollary 4.7 arises in [5] where the following system is studied:

$$
\dot{x}=y, \quad \dot{y}=\theta y-\nabla V(x)
$$

and $x, y \in \mathbf{R}^{n}$. $\theta \in \mathbf{R}$ is taken to be the parameter value and when $\theta=0$ we have a Hamiltonian system. This is a case where the Morse decomposition collapses completely at the special value $\bar{\lambda}=\theta=0$. Nevertheless one can draw weak conclusions about the existence of bounded solutions to the Hamiltonian system by studying the gradient-like systems when $\theta \neq 0$.

\section{REFERENCES}

1. C. C. Conley, Isolated invariant sets and the Morse index, CBMS Regional Conf. Ser. Math., no. 38, Amer. Math. Soc., Providence, R.I., 1978.

2. _ - The gradient structure of a flow. I, IBM Report RC 3932 (\#17806), 1972. 
3. R. Franzosa, Index filtrations and connection matrices for partially ordered Morse decompositions, Thesis, Univ. of Wisconsin, Madison, 1984.

4.

5. K. Mischaikow, Homoclinic orbits in Hamiltonian systems and heteroclinic orbits in gradient and gradient-like systems, LCDS Report \#86-33. 1986.

6. K. Mischaikow and G. Wolkowicz, Predator prey with group defense; A connection matrix approach, preprint.

7. J. Reineck, Connecting orbits in one parameter families of flows, preprint.

8. D. Salamon, Connected simple systems and the Conley index of isolated invariant sets, Trans. Amer. Math. Soc. 29 (1985), 1-41.

9. J. Smoller, Shock waves and reaction diffusion equations, Springer-Verlag, New York, 1983.

Lefschetz Center for Dynamical Systems, Division of Applied MathematICS, BRoWn UNIVERSity, ProvidenCe, Rhode ISLAND 02912

Current address: Department of Mathematics, Michigan State University, East Lansing, Michi$\operatorname{gan} 48824$ 